\title{
LIFELONG LEARNING Y AUTOEFICACIA DEL PROFESORADO
}

\author{
Sciara Salvatore \\ Assegnista di ricerca in Psicologia sociale \\ presso l'Università per Stranieri "Dante Alighieri” di Reggio \\ Calabria (Italia) \\ liana.arcuri@libero.it \\ Arcuri Liana \\ Dottoranda in Educación presso l'Universitat de València (Spagna) \\ liana.arcuri@libero.it \\ Augello Rosanna \\ Assegnista di ricerca in Psicologia dello sviluppo e dell' eeducazione \\ Presso l'Università per Stranieri "Dante Alighieri" di Reggio \\ Calabria (Italia)
}

Fecha de Recepción: 21 Julio 2019

Fecha de Admisión: 25 Septiembre 2019

\section{RESUMEN}

Sentirse profesores eficaces significa creer en las propias capacidades de influir en la prestación de los estudiantes y las modalidades de aprendizaje de los alumnos, incluso los más difíciles o desganados (Guskey y Passaro, 1994). La autoeficacia aumenta la motivación, la cuantía del compromiso empleado, el tiempo dedicado y, en el ámbito laboral, mejora la satisfacción laboral. En el presente trabajo, se ha querido investigar la relación entre la autoeficacia y la formación continua de los profesores, teniendo como objetivo general demostrar que la participación en cursos de actualización, diplomas de perfeccionamiento o master contribuya a aumentar el sentido de eficacia de los profesores. Además, se perseguirán los objetivos de evaluar lo que afecta a la precariedad en el sentido de autoeficacia y analizar las variables que influyen en la autoeficacia percibida por los profesores. A la investigación participaron 225 profesores italianos, que respondieron a través de una suministración on line, a un protocolo de investigación, que comprenda las siguientes escalas de medida: Escala de eficacia personal en el ámbito escolar (Borgogni, Petitta, Steca, 2001), escala de eficacia colectiva percibida en el ámbito escolar (Borgogni, Petitta, Steca, 2001), escala de SelfEfficacy general de Schwarzer, en su adaptación italiana de Lucio Sibilia, Ralf Schwarzer \& Matthias Jerusalem (1995), cuestionario sobre la satisfacción laboral de Weiss et al. (1967), adaptado y validado en italiano por Di Nuovo y Alba (1990), Survey of Perceived Organizational Support de Eisenberg et al. (1986), adaptado y validado en italiano por Battistelli y marianos en 2011, escala del 


\section{LIFELONG LEARNING Y AUTOEFICACIA DEL PROFESORADO}

Compromiso Organizacional y de la implicación en el trabajo de Barbaranelli y Fida (Barbaranelli, Fida, Paciello, Di Giunta, Caprara 2008).

Entre los resultados se desprende que los profesores que han frecuentado más cursos de formación y master en los últimos tres años obtienen puntuaciones significativamente más altas en la escala de Autoeficacia escolar personal respeto a los profesores que no lo han frecuentado; además, los profesores titulares resultan tener una eficacia escolar personal significativamente más alta con respecto a los profesores temporales. Además, del análisis de las regresiones lineales se desprende que las variables que predicen la eficacia escolar personal son la eficacia escolar colectiva, la selfefficacy general y su implicación en el trabajo.

Dado que la eficacia personal está influida también por la eficacia colectiva, en conclusión, parece necesario dirigir tipos de intervención a la escuela centradas sobre las variables de tipo colectivo y organizativo, que mejoren el sentido de pertenencia, la identidad organizativa, la comunicación interna y externa.

Palabras-claves: autoeficacia; formación continua; satisfacción laboral; involucración en el trabajo.

\section{ABSTRACT}

Lifelong learning and teachers' self-effectiviness. Effective teachers should believe in his or her capability to strongly influence students' performance and learning methods, even among those students who may be difficult or unmotivated (Guskey and Passaro, 1994). Self-efficacy enhances motivation, affects the amount of effort and persistence that a person devotes to a task and, on workplace environment, improves job satisfaction. By examining the relationship between selfefficacy and the continuous teacher's training, this paper's overall aim is to demonstrate how attending training courses, post-graduate diplomas or master's degrees helps to increase teachers' sense of efficacy. Moreover, the impact of precariousness on self-efficacy will be analyzed going through the variables influencing teachers' perceived self-efficacy.

This research is based on 225 Italian teachers, who filled out an online research protocol which includes the following measurement scales: School Self-Efficacy Scale (Borgogni, Petitta, Steca, 2001), School Perceived Collective Efficacy Scale (Borgogni, Petitta, Steca, 2001), Schwarzer Generalized Self-Efficacy Scale in its Italian adaptation by Lucio Sibilia, Ralf Schwarzer \& Matthias Jerusalem (1995), Job Satisfaction Questionnaire developed by Weiss et al. (1967) adapted and validated in Italian by Di Nuovo and Alba (1990), Survey of Perceived Organizational Support of Eisenberg et al. (1986) adapted and validated in Italian by Battistelli and Mariani in 2011, Organizational Commitment and Employee Engagement Scale by Barbaranelli and Fida (2004).

Results show that teachers who have attended training courses and masters during the last three years, have obtained significantly higher scores on School Self-Efficacy Scale than teachers who have not attended such courses; furthermore, school's self-efficacy level is considerably higher among tenured teachers than in those who have fixed-term contracts. Moreover, a linear regression analyses reveals that school collective efficacy, generalized self-efficacy and employee engagement are all significant predictors of self-efficacy in school settings. In conclusion, since self-efficacy is also affected by collective efficacy, it is found necessary to focus the learning environment on collaborative and cooperative learning strategies that enhance the sense of belonging, organizational identification, internal and external communication.

Keywords: self-efficacy; continuous teacher training; job satisfaction; employee engagement. 
PSICOLOGIA, INFANCIA Y EDUCACIÓN

\section{INTRODUCCIÓN}

A causa de la globalización, la innovación, la concepción de un mundo sin fronteras, sobre todo en el ámbito laboral, varía el concepto de carrera, de contrato psicológico y, por tanto, el concepto de formación. La carrera se convierte en boundaryless carreer y prevé competencias nuevas, transversales y flexibles. Ya no es suficiente en la nueva concepción de la carrera el know how (saber cómo), sino también el know why (saber por qué) y el know whom (saber para quién) (Noe, Hollenbeck, Gerhart, \& Wright, 2006, p. 18-21). El ambiente de aprendizaje debe ser construido teniendo en cuenta las necesidades, los contenidos a transmitir, pero, sobre todo, de lo vivido y los sentimientos de las personas comprometidas en este. La formación debe tener en cuenta los contenidos y los comportamientos, pero debe privilegiar lo vivido y los sentimientos de las personas y por eso se debe alejar de un entorno que prevé un formador diferente de los demás que observa todos y es percibido como controlador (Salvetti, 2013, p. 227-230).

El mundo contemporáneo, caracterizado por constantes transformaciones e innovaciones en todos los campos, requiere hombres que no sufran pasivamente los acontecimientos o que reaccionen a ellos según las modalidades ya aprendidas, sino hombres que sean capaces de seleccionar la solución y ponerla en práctica para el logro de sus objetivos. No debemos hablar, por tanto, de un hombre agente activo, sino de hombre agente proactivo que tiene el control de su vida (locus of control interno). Bandura (2000), a este respecto, introduce el concepto de "agentividad" entendiendo con este término la habilidad del hombre de intervenir sobre la realidad para hacer que sucedan los acontecimientos por él fijados (Bandura, 2000). La percepción de autoeficacia por parte del docente es especialmente importante por lo que concierne la selección de los objetivos, los contenidos, las competencias y las capacidades que se pretenden desarrollar, y también, para la definición y organización de las modalidades de aprendizaje y, por tanto, de identificación de las estrategias adecuadas para mantener un alto nivel de motivación. Bandura sostiene que es la autoeficacia que determina el comportamiento y no viceversa, pues el profesor debe desarrollar las creencias de autoeficacia los alumnos utilizando la enseñanza personalizada para reducir de este modo las comparaciones sociales desalentadoras; estructurar actividades didácticas sobre bases operativas y favorecer el tutoring activo entre estudiantes, repartiendo las actividades complejas en sub objetivos para tener feedback positivos. Todo esto demuestra la centralidad del papel del docente y de su autoeficacia percibida. La autoeficacia del docente es central en las relaciones entre escuela, estudiantes y familia, es fundamental para mediar entre el mundo y la cultura. La autoeficacia los profesores es, por tanto, un concepto con muchas facetas y que tiene implicaciones muy amplias si se piensa a quién y cuanto se ve involucrado. Muchos autores han dado una definición. Bermann, por ejemplo, considera que la autoeficacia se puede definir como el grado en que el docente cree ser capaz de influir en las prestaciones de los estudiantes (Bermann, McLaughlin, Bass, Pauly, \& Zellman, 1977, p. 184-187). Otra definición importante es la que explicita la autoeficacia del docente como la capacidad de tener influencia sobre la motivación de todos los estudiantes, especialmente de aquellos desganados o desfavorecidos que, como veremos, son determinantes para determinar el grado de autoeficacia (Guskey, \& Passaro, 1994, p. 627-643).

Considerando que una alta autoeficacia está asociada a la adopción de innovaciones (Fuchs, Fuchs, \& Bishop, 1992, p. 70-84), a las motivaciones y al rendimiento escolar de los estudiantes (Multon, Brown, \& Lent, 1991, p. 30-38) a bajos niveles de estrés (Parkay, Greenwood, Olejnik, \& Proller, 1988, p. 13-22), de los resultados de las investigaciones que hemos citado podemos esquematizar las características de los profesores que tienen un alto nivel de autoeficacia y de los que lo tienen bajo. Los profesores con alto nivel de autoeficacia se centran más en las actividades didácticas y las hacen propias buscando un intercambio continuo con el grupo clase para aumentar sus 


\section{LIFELONG LEARNING Y AUTOEFICACIA DEL PROFESORADO}

conocimientos, no en forma estática y estéril, no temen la heterogeneidad de las clases y consiguen relacionarse eficazmente con todos los alumnos y también apoyar y ayudar a los alumnos discapacitados, proporcionan a los alumnos gratificaciones por sus éxitos reforzando así su motivación, suelen utilizar los refuerzos positivos con sus alumnos precisamente porque creen en su trabajo, durante las horas que pasan en clase logran tener una relación serena con los alumnos sin sentir la necesidad de recurrir a medios coactivos y autoritarios, el tiempo transcurrido en clase se caracteriza por "enseñanza activa" y no por la mera adopción de estrategias de transmisión, asistencial y restrictiva, con respecto a los colegas que tienen un bajo nivel de autoeficacia son más entusiastas respecto a su trabajo y aman su trabajo. Los profesores que tienen un bajo nivel de autoeficacia se rinden fácilmente si sus alumnos no obtienen los resultados en los plazos previstos, dedican una parte del tiempo a actividades no estrictamente escolares sino recreativas, no son capaces de gestionar la clase y para resolver los problemas de comportamiento no recurren al refuerzo de interés y la motivación, sino a un control ejercido a través de normas estrictas y coercitivas, no hallando $n$ sí el modo de suscitar interés en los alumnos están acostumbrados a utilizar premios y castigos, se dedican únicamente a la disciplina objeto de su enseñanza, muchos de ellos, no sintiéndose gratificados, cambiarían gustosamente trabajo. Podemos afirmar que el profesor para ser eficaz debe tener los requisitos indicados por Meyer (2007) que le permiten construir su propia autoeficacia: tener una buena preparación profesional, didáctica e pedagógica; saber estructurar de forma clara una clase; saber utilizar una comunicación estimulante; saber utilizar metodologías didácticas. Entornos relacionales difíciles y una general desconfianza en las componentes de la organización escolar, pueden representar disuasiones capaces de contrarrestar y desalentarlos también a los profesores más preparados. De hecho, junto a la eficacia personal, la percepción del contexto, se revela una importante variable capaz de mediar la influencia de la primera sobre la eficacia colectiva. La encuesta internacional sobre la enseñanza y aprendizaje (TALIS) de la OCSE (OECD, 2016) muestra los elementos que pueden influir en una enseñanza eficaz: formación inicial y desarrollo profesional, feedback recibido respecto a las actividades de enseñanza realizadas, clima en las clases y en las escuelas donde se trabaja, grado de satisfacción con respecto a la propia profesión y percepción de las propias capacidades profesionales. En la encuesta se revela también la importancia de la formación inicial: Ios profesores cuya formación inicial incluía contenidos y elementos de pedagogía y práctica didáctica dentro de las clases para las materias específicas que enseñan en la actualidad, hablan de sentirse mejor preparados para su trabajo de profesores respecto a los colegas que no han recibido este tipo de formación inicial.

Por lo tanto, los profesores necesitan aprender las competencias y conocimientos de según la lógica de lifelong learning, la formación sistémica y de boundaryless learning. La educación y la formación en la óptica del lifelong learning es el mejor, si no el único, modo para afrontar el cambio que se está realizando.

\section{LA INVESTIGACIÓN}

\section{Objetivos e hipótesis de investigación}

La investigación que ha sido realizada ha tenido como objetivo principal demostrar que la participación en cursos de actualización, diplomas de perfeccionamiento o Master, que den la posibilidad de aumentar los conocimientos disciplinarios y las competencias para la gestión de la actividad didáctica, contribuye a aumentar el sentido de eficacia de los profesores.

En concreto, se persiguen los siguientes objetivos: investigar cómo varía el nivel de autoeficacia de los profesores implicados en cursos de formación; evaluar cuanto influye la precariedad en el sentido de la autoeficacia; explorar si el nivel de autoeficacia sufre variaciones dependiendo de 
que la contratación sea determinada por un modelo profesional (cursos de licenciatura, SISSIS, TFA) o por un modelo meritocrático (concurso); analizar las variables que influyen en la autoeficacia percibida por los profesores.

Las hipótesis formuladas son:

1. La formación continua del profesorado aumenta su sentido de autoeficacia y, por tanto, una mayor cantidad de experiencias formativas (cursos de perfeccionamiento, Master, etc.) conduce a una mayor autoeficacia;

2. Ser precarios repercute negativamente sobre el sentido de autoeficacia de los profesores.

3. Convertirse en titular gracias a la cantidad de títulos formativos y habilitadores o gracias a la superación de un concurso no tiene una influencia sobre el nivel de autoeficacia, visto que la primera condición hace experimentar al profesor el éxito gracias a su formación y profesionalidad y la segunda condición implica iniciar su carrera de profesor a tiempo indefinido a partir de un modelo meritocrático (superación de un concurso);

4. La eficacia personal escolar de los profesores es influenciada por la dimensión colectiva de la eficacia escolar y por el apoyo organizacional percibido, es decir, que los profesores se sienten más eficaces como profesores si reciben también la escuela donde trabajan como capaz de enfrentarse bien a las dificultades y si sienten el apoyo por parte de la Organización escolar.

\section{Participantes}

A la investigación participaron 225 profesores, pero siete protocolos han sido invalidados, por faltar respuestas o por respuestas evidentemente no verdaderas, por lo cual la muestra está compuesta por 218 sujetos. La suministración se produjo on line y el protocolo de investigación ha sido introducido en algunos sitios web específicos sobre el mundo de la escuela. Al cuestionario se acedía mediante un link, precedido por una breve presentación y una invitación a dar su contribución a una investigación sobre la escuela y los profesores.

La mayoría de los profesores es de género femenino (85,8\%), mientras que la parte restante son varones; esta proporción refleja, sin embargo, la prevalencia femenina que existe en el mundo de la escuela. La muestra es heterogénea en cuanto a la edad, que varía de un mínimo de 26 años a un máximo de 60 con una edad media de 40,50 años, y una desviación estándar de 7,69 años. La misma heterogeneidad la encontramos en la variable "antigüedad", que varía incluso de 1 a 35 años, con una media de 9,89 años y una desviación estándar de 7,43 años. La mayor parte de los profesores que han participado en la investigación declara residir en el Sur de Italia $(62,8 \%)$, seguidos por los residentes en el Norte $(21,1 \%)$; la cifra más baja es la de los profesores del Centro de Italia $(16,1 \%)$.

La casi totalidad de los profesores encuestados, el 95\%, frecuentó en los últimos 3 años master 0 cursos de perfeccionamiento. El $61 \%$ de los docentes encuestados no trabaja en la misma ciudad donde vive, un dato que hace reflexionar sobre una de las dificultades actuales de la clase docente, obligada a desplazarse en la sede donde es llamada a trabajar, una sede que también puede estar varios kilómetros lejos de casa.

\section{Instrumentos}

El protocolo de investigación comprende las siguientes escalas de medida:

Escala de eficacia personal en el ámbito escolar (Borgogni, Petitta, Steca, 2001 pp. 157-171), formada por 12 ítem que miden las convicciones de los docentes relativas a su capacidad de hacer frente a las demandas del ambiente y a superar con éxito las situaciones difíciles a la que se enfrentan en la actividad escolar. Es una escala tipo Likert de 7 posiciones (de $1=$ muy en desacuerdo a 7 = muy de acuerdo). Ejemplos de ítem: Sé cómo implicar en las distintas actividades previstas desde 


\section{LIFELONG LEARNING Y AUTOEFICACIA DEL PROFESORADO}

mi enseñanza también los alumnos más resistentes y difíciles. Soy capaces de superar todas las dificultades que encuentro en la realización de los objetivos didácticos. Soy capaz de hacerme apreciar como docente por todas las familias.

Escala de eficacia colectiva percibida en el ámbito escolar (Borgogni, Petitta, Steca, 2001), constituida por 9 ítem que miden las convicciones de los docentes relativas a la capacidad de su escuela de hacer frente a las demandas del ambiente y de superar con éxito las situaciones difíciles. Es una escala tipo Likert de 7 posiciones (de $1=$ muy en desacuerdo a $7=$ muy de acuerdo). Ejemplos de ítem: nuestra escuela es capaz de ejercer una importante acción de promoción y desarrollo también en el territorio. Nuestra escuela sabe transmitir al exterior una imagen positiva. Nuestra escuela es capaz de realizar intervenciones eficaces para prevenir el abandono escolar.

Escala de Self-Efficacy general de Schwarzer, en su adaptación italiana de Lucio Sibilia, Ralf Schwarzer \& Matthias Jerusalem (1995), constituida por 10 ítem que evalúan el sentido general de autoeficacia. É una escala Likert que va desde $1=$ Para nada cierto 2 a $4=$ totalmente cierto. Ejemplos de ítem: Puedo siempre a resolver problemas difíciles si lo intento lo suficientemente en serio. Si alguien me contradice, puedo encontrar el modo o el sistema para obtener lo que quiero. Para mí es fácil atenerme a mis intenciones y alcanzar mis objetivos.

Cuestionario sobre la satisfacción laboral de Weiss et al. (1967), adaptado y validado en italiano por Di Nuovo y Alba (1990), compuesto por 11 ítem que señalan el grado de satisfacción laboral. La escala Likert está a 4 puntos. Ejemplos de ítem: el trabajo que cumplo corresponde a mis expectativas. Los Superiores dan los reconocimientos cuando los operadores realizan bien su trabajo. Me encuentro bien en la relación con los compañeros de trabajo.

Survey of Perceived Organizational Support de Eisenberg (Santos \& Eisenhardrt, 2005), adaptado y validado en italiano por Battistelli y Mariani en 2011, constituido por 8 Ítem de respuesta múltiple en escala Likert de 5 puntos, es un cuestionario sobre la percepción por parte de los profesores de cuanto la organización se muestre atenta a evaluar y valorar tanto las contribuciones recibidas por los propios trabajadores como su estado de bienestar. Ejemplos de ítem: Si tengo un problema, mi escuela está dispuesta a ayudarme. Mi escuela se preocupa realmente por mi bienestar. Mi escuela perdonaría un error mío de buena fe.

Escala del Compromiso Organizativo y de la implicación en el trabajo de Barbaranelli y Fida (2004). Se trata de dos escalas que miden el compromiso en la consecución de los objetivos comunes y la implicación emocional en el trabajo. La escala Likert está a 4 puntos. Ejemplos de ítem: Renuncio gustosamente a una parte de mi tiempo libre para llevar a cabo mis compromisos laborales. Para terminar un trabajo me quedo espontáneamente más allá del horario fijado, aunque no venga pagado por ello. Estoy muy involucrado personalmente en mi trabajo. Mi escuela tiene para mí un gran significado personal. Siento un fuerte sentimiento de pertenencia a esta escuela.

Además, el protocolo de investigación comprende una amplia ficha socio-demográfica, que ha permitido detectar las siguientes variables: género, edad, estado civil, región de pertenencia, grado de escuela en la que enseña, disciplina enseñada, antigüedad, modalidades de contratación, duración del empleo precario, situación contractual (titular o no titular) desde cuántos años, tipo y cantidad de experiencias formativas, las necesidades formativas, estar en sede/fuera de la sede/viajeros, la intención de cambiar de trabajo y sede de trabajo.

\section{Análisis de Datos}

Ha sido posible realizar diferentes tipos de análisis:

Estadísticas descriptivas: medias, frecuencias, frecuencias porcentuales;

Análisis de la varianza, con el fin de medir las diferencias significativas según las variables inves- 
tigadas (tipo y cantidad de experiencias formativas, modalidades de contratación, años de antigüedad, etc.).

Análisis de las correlaciones, para medir las relaciones entre las variables investigadas;

Análisis de las regresiones, para evaluar la influencia de las variables independientes sobre la dependiente.

Además, ha sido evaluada la fiabilidad de las escalas utilizadas, mediante el cálculo del Alpha de Cronbach, que quedó elevado 0 , de todas formas, satisfactorio para todas las escalas introducidas en el protocolo de investigación.

\section{RESULTADOS}

Los resultados de la investigación han confirmado las hipótesis de investigación (la última de forma parcial), estimulando la reflexión también hacia direcciones que no se habían supuestos durante la fase inicial y proporcionando ideas para investigaciones futuras.

Casi todos los docentes, el 92,2\%, consideran su formación personal adecuada para ocupar su posición laboral actual. La muestra no parece, por lo tanto, expresar una fuerte necesidad formativa, sino la conciencia de tener ya un cierto bagaje de experiencias formativas, testimoniado por el elevado número de cursos frecuentados.

Nuestra muestra tiene una eficacia personal percibida en el ámbito escolar superior a la media de la muestra reglamentaria. Al contrario, la eficacia colectiva percibida en el ámbito escolar es bastante inferior a la norma.

Choca la diferencia entre la puntuación (elevada) relativo a la eficacia personal y la puntuación (bastante baja) de la eficacia colectiva. Esto significa que los profesores se sienten buenos y competentes a nivel personal, pero perciben su escuela y/o su grupo de trabajo como inadecuado y no siempre capaz de afrontar las dificultades.

En confirmación de nuestra hipótesis de partida, los profesores titulares resultan tener una eficacia escolar personal significativamente más alta con respecto a los profesores que tienen cargos por tiempo determinado.

También la hipótesis de que la cantidad de cursos de formación tiene una influencia sobre la autoeficacia es confirmada: en efecto, por los datos se desprende que los profesores que han frecuentado más cursos de formación y master en los últimos tres años obtienen puntuaciones significativamente más altas en la escala de Autoeficacia escolar personal respeto a los profesores que no los han frecuentado.

La tercera hipótesis fue confirmada porque no se constató ninguna diferencia significativa en cuanto a la autoeficacia entre los profesores que han sido contratados a través de concurso y aquellos que entraron titulares a través de una clasificación. Evidentemente, también quién no ha superado un concurso, considera tener 'merecida' la contratación a tiempo indeterminado, ya sea porque la puntuación en la clasificación es dado en parte por los títulos formativos, tales como cursos, master y habilitaciones, sea porque los años de servicio realizados como precarios probablemente dan la sensación de que la propia experiencia debe ser reconocida mediante la adquisición de puntuaciones y la consecuente y posterior contratación, también con respecto a quién, aun superando las pruebas de un concurso, quizás no tenga la competencia profesional derivada de una (más 0 menos larga) experiencia real en el aula.

Los profesores que quieren cambiar de trabajo tienen puntuaciones significativamente más bajas respecto a quien no quiere cambiar de trabajo en las escalas Eficacia Escolar Personal, Eficacia general, Satisfacción laboral y Participación en el trabajo, mientras que aquellos que desean cambiar de escuela obtienen puntuaciones significativamente más bajas que los compañeros que no 


\section{LIFELONG LEARNING Y AUTOEFICACIA DEL PROFESORADO}

quieren cambiar de escuela, en las escalas (menos vinculadas a la esfera individual y más ligadas al contexto) Eficacia Escolar Colectiva, Satisfacción Laboral, Implicación en el Trabajo y Apoyo Organizativo Percibido.

Del análisis de las correlaciones surge una correlación significativa entre los años de titular y el compromiso organizativo; en concreto al aumentar de los años de titular también aumenta el compromiso organizativo. Pasando a las correlaciones entre las escalas, las correlaciones más altas resultan ser las entre la Eficacia Escolar Personal y de la self-efficacy General $\left(r=, 538^{* *}\right)$, por lo cual sentirse personas eficaces en general se relaciona significativamente con el sentirse profesores eficaces, entre el Compromiso Organizativo e Implicación en el Trabajo $\left(r=, 527^{\star \star}\right)$, por tanto, más nos sentimos implicados en el trabajo y más nos empeñamos, y entre el Compromiso Organizativo y Apoyo Organizativo percibido $\left(r=, 517^{\star *}\right)$, por lo cual nos comprometemos en el trabajo cuanto más percibimos el apoyo de los demás, en cierto sentido, nos comprometemos en la medida en que sentimos también el compromiso de los demás y no nos sentimos solos.

La satisfacción laboral se relaciona más con la eficacia escolar colectiva $\left(r=, 434^{\star *}\right)$, y confirma que cada profesor se siente satisfecho de su trabajo si percibe positivamente la escuela donde desarrolla su servicio, esto significa que la satisfacción laboral puede cambiar cambiando la escuela. Finalmente, para alcanzar el último objetivo de la investigación y confirmar la cuarta hipótesis, hemos efectuado un análisis de las regresiones de tipo lineal, poniendo la eficacia escolar personal como variable dependiente y todas las demás como variables independientes.

Las variables que predicen la eficacia escolar personal son la eficacia escolar colectiva, la selfefficacy general y su implicación en el trabajo, confirmando sólo en parte nuestra hipótesis de partida. En efecto, el apoyo organizativo percibido no constituyó una variable predictiva de autoeficacia, probablemente porque el primero se atribuye únicamente a los demás, mientras que la dimensión de autoeficacia sería sentida como más puramente personal. A pesar de ello, esta última estaría influida por la autoeficacia escolar colectiva, probablemente porque para poder ejercer plenamente su papel de profesor, se advierte como necesaria la pertenencia a una organización escolar que tenga una imagen positiva, que sea eficiente, a la vanguardia, apreciada, capaz de hacer frente a las dificultades y las demandas del contexto (familias, comunidades, territorio).

En conclusión, por los resultados se desprende, por varios frentes, la importancia atribuida a los factores contextuales además que a los estrictamente individuales. En primer lugar, por lo que respecta a los aspectos más personales, los profesores sienten poseer una buena formación de partida, testimoniada entre otras cosas por el amplio número de títulos y habilitaciones (Master, cursos, SISSIS, TFA...) poseídos; a partir de esto, los profesores obtienen puntuaciones elevadas en la escala de auto-eficacia escolar personal, demostrando que se perciben como los buenos profesores, capaces de afrontar las dificultades y de hacerse apreciar por los alumnos, los padres y el directivo. En consonancia con estos datos, la mayor parte de los profesores no querrían cambiar de trabajo, demostrando una elevada motivación para desempeñar la profesión docente que, probablemente, es aquella que responde a sus aspiraciones y aptitudes.

La primera hipótesis de investigación resulta confirmada por el dato que ve los profesores con una mayor cantidad de experiencias formativas, en los últimos tres años, obtener puntuaciones significativamente más elevadas en la escala de Autoeficacia escolar personal respeto a los colegas que han realizado un menor número de cursos y master en los últimos tres años. Este dato pone de relieve y demuestra la importancia de una formación continua, realizada durante su carrera de docente, que respalde su trabajo, sometido a continuos desafíos y sujeto a constantes cambios. Con un adecuado bagaje formativo, en continua actualización, el profesor se puede percibir como eficaz y capaz de afrontar los retos diarios de su trabajo, respecto a las dificultades de los alumnos, a la relación 
con las familias, a la organización escolar, los cambios normativos, las nuevas metodologías didácticas, y así sucesivamente. Confirmando nuestra hipótesis de partida, los datos muestran que los profesores titulares tienen una eficacia escolar personal más elevada con respecto a los profesores a tiempo determinado. Este resultado expresa la diferencia que existe entre profesores titulares y sus suplentes 0 contratados a tiempo determinado. A menudo en la escuela, los profesores precarios son considerados 'de paso', o aún peor, 'los últimos llegados', a menudo no tienen cargos adicionales, no vienen incluidos en proyectos extracurriculares y raramente ejercen funciones de soporte para el directivo escolar, resultando en última instancia poco valorizados y poco integrados dentro de la Organización escolar.

Comenzando a considerar los factores de tipo contextual-organizativo, se desprende que, en primer lugar, un porcentaje mucho más elevado con respecto a los profesores que quieren cambiar de trabajo, cambiaría escuela en la que trabaja, mostrando una intención de querer trasladarse, porque no se encuentra bien en términos de clima escolar, o porque la sede de la escuela se encuentra alejada de la ciudad de residencia. Esta hipótesis es confirmada por el hecho de que a querer cambiar de escuela son principalmente los docentes fuera de la sede y viajantes.

En segundo lugar, frente a puntuaciones más altos de la media obtenidos por los participantes en la escala de Autoeficacia escolar personal, los profesores obtienen puntuaciones medias muy inferiores a la media de la muestra normativa en la escala de Autoeficacia escolar colectiva. Esto significa que los profesores, aunque sintiéndose capaces y competentes y no queriendo cambiar de trabajo, perciben, al contrario, la escuela en la que trabajan o su grupo de trabajo como inadecuado y no siempre capaz de afrontar los problemas y los imprevistos. Coherente a este resultado aparece la intención de muchos de querer cambiar de escuela.

\section{CONCLUSIONES}

Estos resultados orientan la reflexión hacia la necesidad de una intervención en la escuela no tan centrado en competencias de los distintos profesores, sino sobre las variables más de tipo colectivo y organizativo, que mejoren el sentido de pertenencia, la identidad organizativa, la comunicación interna y externa y todos aquellos aspectos que puedan mejorar la autoeficacia escolar colectiva.

La importancia de esta última variable es confirmada por la alta correlación que hemos encontrado con la satisfacción laboral y la diferencia significativa entre los profesores que quieren cambiar de escuela o menos en la escala de autoeficacia escolar colectiva. Por tanto, la autoeficacia escolar colectiva afecta a la satisfacción laboral y a la intención de cambiar de escuela, una importante indicación operativa que emerge de nuestra investigación es la de intervenir en la mejora 0 , mejor dicho, en la construcción de la percepción de una buena eficacia escolar colectiva.

\section{REFERENCIAS BIBLIOGRÁFICAS}

Bandura, A. (2000). Autoefficacia: teoria e applicazioni. Trento: Erikson Editore.

Barbaranelli, C., Fida, R., Paciello, M., Di Giunta, L., Caprara, G. V. (2008). Assessing personality in early adolescence through self-report and other-ratings: A multitrait-multimethod analysis of the BFQ-C. Personality and Individual Differences, 44, 876-886.

Bermann, P., McLaughlin, M., Bass, G., Pauly E., Zellman G. (1977). Federal programs supportino educational change. Vol. VII: Factor affecting implementation and continuation. Report R1589/7- HEW. Santa Monica, CA: The Rand Corporation.

Borgogni, L., Pettita, L., Steca, P. (2001). Efficacia personale e collettiva nei contesti organizzativi. In G.V. Caprara (Ed.). La valutazione dell'autoefficacia. Interventi e contesti culturali. Trento: Erickson. 
Fuchs, L.S., Fuchs, D., Bishop, N. (1992). Instructional adaptation for students at risk. Journal of Educational Research, 86, 70-84.

Guskey, T., Passaro, P. (1994). Teacher efficacy: A study of construction dimensions. American Educational Research Journal, 311, p. 627- 643.

Meyer, H. (2007). Was ist guter unterricht? Berlino: Cornelsen Scriptor.

Multon, K., Brown, S., Lent, R. (1991). Relation of self-efficacy beliefs to academic outcomes: A meta- analytic investigation. Journal of Counseling Psychology, 38.

Noe, R.A., Hollenbeck, J.R., Gerhart, B., Wright, P.M. (2006). Gestione delle risorse umane. Milano: Apogeo.

OECD (2016). Risultati TALIS 2013: una prospettiva internazionale sull'insegnamento e sull'apprendimento. OECD Publishing. Retrieved September 3, 2016, from http://dx.doi.org/10.1787/9789264251694-it

Parkay, F. W., Greenwood, G., Olejnik, S., Proller, N. (1988). A study of the relationship among Teacher burnout 241 teacher efficacy, locus of control, and stress. Journal of Research and Development in Education, 21(4), 13-22.

Salvetti, F. (2013). Ambienti. In F. Amicucci, G. Gabrielli, (Ed.). Boundaryless learning. Nuove strategie e strumenti di formazione. Milano: Franco Angeli.

Santos, F.M., \& Eisenhardrt, K.M. (2005). Organizational boundaries and teories of organization. Organization Science, n. 16, 491- 508.

Sibilia, L., Schwarzer, R., Jerusalem, M., (1995) Italian adaptation of the General Self-Efficacy Scale: self-efficacy generalized. Procedia - Social and Behavioral Sciences.

Weiss, J.M. (1991). Stress-induced depression: critical neurochemical and electrophysiological changes. In J. Madden, (ed.). Neurobiology of learning, emotion and affect. New York: Raven, pp. 123-154. 